REVISTA DE ESTUDIOS E INVESTIGACIÓN

EN PSICOLOGÍA Y EDUCACIÓN

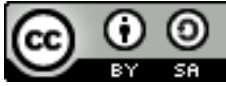

ISSN: 1138-1663; elSSN: 2386-7418

UDC / UMinho

2021, Vol. 8, No. 2, 242-260.

DOI: https://doi.org/10.17979/reipe.2021.8.2.8692

\title{
Bienestar emocional y resiliencia en profesionales de la educación social durante el confinamiento por la COVID-19
}

\section{Emotional well-being and resilience of social educators during the COVID-19 lockdown}

\begin{abstract}
Julio Rodríguez-Rodríguez (D) https://orcid.org/0000-0001-9202-2589
Departamento de Métodos de Investigación y Diagnóstico en Educación, Facultad de Educación, Universidad de Barcelona: https://www.ub.edu/portal/web/educacion Daniel Ortega-Ortigoza (iD https://orcid.org/0000-0002-8581-4833

Departamento de Pedagogía Aplicada, Facultad de Ciencias de la Educación, Universidad Autónoma de Barcelona: https://www.uab.cat/web/facultad-de-ciencias-de-la-educacion1345747134964.html
\end{abstract}

Ainoa Mateos (D) https://orcid.org/0000-0002-1159-9966

Departamento de Métodos de Investigación y Diagnóstico en Educación, Facultad de Educación, Universidad de Barcelona: https://www.ub.edu/portal/web/educacion

Barcelona, Cataluña - España

Correspondencia relativa a este artículo: Julio Rodríguez-Rodríguez - julio.rodriguez.ro@ub.edu 


\title{
Resumen
}

La pandemia de COVID-19 y las medidas de confinamiento resultantes han impactado en la salud física y mental de la población de diferentes maneras. Como profesionales esenciales, los educadores sociales que trabajan en centros de atención residencial para niños y jóvenes han tenido que hacer frente a presiones adicionales como las medidas de confinamiento domiciliario y tener que continuar su trabajo en circunstancias excepcionales. Para evaluar el impacto de las medidas de cierre en el bienestar emocional y la resiliencia de los educadores sociales, se realizó un estudio exploratorio ex post facto basado en una muestra de 72 educadores sociales $(30,6 \%$ hombres y $69,4 \%$ mujeres) que trabajan en centros residenciales de protección de la infancia en Cataluña. La encuesta recogió datos sobre el bienestar emocional (PANAS y SWLS) y la resiliencia (RS-14), y también los datos sociodemográficos de los participantes. Los resultados muestran valores similares en relación con el bienestar emocional y la resiliencia, sin diferencias significativas entre las categorías sociodemográficas. Se encontró una correlación positiva entre el equilibrio afectivo, la satisfacción vital y la resiliencia. Las conclusiones del estudio ponen de manifiesto la necesidad de apoyar el bienestar emocional de los educadores sociales promoviendo su capacidad de resiliencia, teniendo en cuenta la ya de por sí compleja tarea de atención socioeducativa a la infancia y adolescencia en acogimiento residencial, y la dificultad adicional del confinamiento.

Palabras clave: afectividad; satisfacción con la vida; afrontamiento; pandemia; acogimiento residencial

\begin{abstract}
The COVID-19 pandemic and resulting lockdown measures have impacted on the physical and mental health of the population in different ways. As essential professionals, social educators working in residential care centres for children and young people have had to deal with additional pressures such as lockdown measures and having to continue their work under exceptional circumstances. To assess the impact of lockdown measures on social educators' emotional well-being and resilience, an ex post facto exploratory study was conducted based on a sample of 72 social educators (30.6\% men and $69.4 \%$ women) working in residential childhood protection centres in Catalonia. The survey collected data on emotional well-being (PANAS and SWLS) and resilience (RS-14), and also the participants' sociodemographic details. The results show similar values in relation to emotional well-being and resilience, with no significant differences between sociodemographic categories. A positive correlation was found between affective balance, life satisfaction and resilience. The conclusions of the study highlight the need to support the emotional well-being of social educators by promoting their capacity for resilience, taking into account the already complex task of the socio-educational care of children and adolescents in residential care, and the additional difficulty of lockdown.
\end{abstract}

Keywords: affectivity; life satisfaction; coping; pandemic; foster care 
La pandemia por la COVID-19 está teniendo unas repercusiones sin precedentes, amenazando la salud mundial (Walker et al., 2020), propiciando una crisis global, con un profundo impacto en la salud pública, la sociedad y la economía (Banco de España, 2020; Fondo Monetario Internacional, 2021), y se han visto damnificados todos los sectores: primario, secundario y terciario (Nicola et al., 2020). A esto, se añade la declaración del estado de alarma por parte del Gobierno de España (RD 463/2020) y las medidas temporales de carácter extraordinario, como el confinamiento domiciliario de la población y el cese de las actividades no esenciales.

Durante el estado de alarma, solamente las profesiones esenciales estaban autorizadas a trabajar de forma presencial (RD 10/2020). Las y los educadores sociales en el ámbito residencial de protección a la infancia y la adolescencia han sido uno de esos colectivos esenciales. Es un perfil profesional específico y muy especializado (De Juanas et al., 2014; Estévez et al. 2017; Fernández del Valle, 2009), y complejo en relación con la acción socioeducativa que desarrolla y el encargo institucional que asume (García y Sánchez, 2010; Lázaro et al., 2020; Montserrat y Melendro, 2017; Vallés, 2011). Su tarea conlleva una serie de riesgos psicosociales: estrés, inseguridad, burnout profesional, insatisfacción laboral y problemas de salud (Fernández del Valle et al., 2007; Jenaro et al., 2007; Navarro et al., 2018); agresiones físicas en el lugar de trabajo (Col-legi de Educadores i Educadors Socials de Catalunya, [CEESC, 2019]), y aumento en la carga laboral, o falta de recursos y formación adecuadas (Martínez-Pérez y Lezcano, 2020). En relación con la COVID-19, más del $80 \%$ de la profesión ha seguido trabajando. Emocionalmente, el estrés, la ansiedad, la soledad y la tristeza son los elementos que se han evidenciado con más impacto. $\mathrm{Y}$ es que más del $90 \%$ de las personas atendidas por las y los profesionales socioeducativos se ha visto afectadas por esta crisis sanitaria (CEESC, 2020). Esto da una cuenta de la sobrecarga emocional que han tenido que afrontar las personas usuarias y profesionales durante la evolución de la pandemia y el confinamiento domiciliario.

Frente a la adversidad que ha representado la pandemia y el confinamiento, las personas han podido desplegar diferentes estrategias de afrontamiento (Lazarus y Folkman, 1986), con independencia del resultado esperado (Campos et al., 2004; Fernández-Abascal y Palmero, 1999). En este afrontamiento, los afectos positivos y negativos facilitan o interfieren la capacidad de respuesta (Limonero et al., 2012). Además, los afectos y la satisfacción con la vida son los componentes del bienestar emocional (Atienza et al., 2000; Castro, 2009).

El bienestar se ha entendido como una valoración subjetiva y positiva que una persona hace sobre su vida (Ryff y Singer, 2000). Identificar el propio bienestar representa cierta 
complejidad, desde un punto de vista científico (Vázquez et al., 2009), existiendo un debate entre dos perspectivas filosóficas: hedonismo y eudaimonia. Para la primera, el bienestar implica la presencia de afectos positivos y la ausencia de negativos. Para la segunda, el bienestar es una forma de vida plena o de realización del potencial humano. Conceptos básicos del bienestar hedónico serían el placer, los afectos positivos y negativos, el balance afectivo o la satisfacción vital. $Y$ entre los instrumentos que se utilizan para medirlo se encuentran la escala de afecto positivo y negativo (PANAS) de Watson et al. (1988) y la escala de satisfacción con la vida (SWLS) de Diener et al. (1985). Existen evidencias acerca de los componentes del bienestar subjetivo: uno de carácter emocional (afectos positivos y negativos) y otro de carácter cognitivo (satisfacción con la vida) (Atienza et al., 2000; Castro, 2009). La satisfacción con la vida es un proceso cognitivo que implica comparar las circunstancias en las que se encuentra el individuo y aquello considerado un estándar adecuado (Diener et al., 1985). Aunque están relacionados, se recomienda evaluar por separado afectos y satisfacción con la vida (Atienza et al., 2000; Reppold et al., 2019).

El bienestar subjetivo es, para algunas autoras y autores, un componente interpersonal de la resiliencia (Vanistendael, 2018), constituyendo un factor protector frente a las adversidades.

Como se ha señalado, verse afectado por una adversidad grave, como han sido la COVID19 y el confinamiento domiciliario, ha podido afectar la salud mental de las personas. A su vez, estas adversidades pueden hacer aflorar procesos resiliencia, como elemento protector de la salud mental, como han documentado Robles-Bello et al. (2020). Aunque no existe una definición universal del concepto (Knight, 2007) y se den algunos matices según las autoras y autores que la conceptualizan (Vera et al., 2008), la resiliencia puede entenderse como un proceso de cambio y transformación en las formas de entenderse a uno mismo, a los demás y la vida (Manciaux et al., 2003; Rutter, 2012). En esta pluralidad de definiciones y elementos que la conforman, y para abarcar su complejidad (Sisto et al., 2019) se distinguen cinco grandes grupos o áreas implicadas en la resiliencia: la habilidad para la recuperación frente a las adversidades, un funcionamiento que caracteriza a la persona, la capacidad de recuperarse y desarrollar los propios recursos, un proceso dinámico que evoluciona con el tiempo, y la adaptación positiva a los acontecimientos vitales. Este enfoque individualista de la resiliencia se ha enriquecido con perspectivas ecológicas (Vaquero et al., 2014; Ungar, 2011), enfatizando los determinantes ambientales. Por ello, diversas autoras y autores entienden la resiliencia como un proceso que, desde un enfoque ecológico, implica al sujeto, la familia, la comunidad y la sociedad como elementos significativos interrelacionados (Infante, 2001; Vanistendael, 2018). 
Así, teniendo en cuenta lo expuesto anteriormente, identificar el bienestar emocional y la resiliencia de las personas que tienen como tarea la atención socioeducativa y el cuidado de otras personas es un aspecto relevante en la salud mental de esta profesión, como ponen de manifiesto Sánchez-Teruel et al. (2021) en el ámbito del cuidado domiciliario. Este bienestar y resiliencia pueden modular la calidad de la intervención, especialmente en el ámbito de la protección a la infancia. Como se ha apuntado, este bienestar ha podido verse comprometido por el impacto de la pandemia y el posterior confinamiento domiciliario si no han podido desplegarse elementos protectores. La finalidad de esta investigación ha sido indagar si el impacto de la COVID-19 y el confinamiento domiciliario ha podido comprometer el bienestar emocional y la resiliencia de un grupo de educadoras y educadores sociales que trabajan en el ámbito residencial de protección a la infancia y adolescencia en Cataluña. Los objetivos específicos fueron: 1) determinar el grado de bienestar emocional y resiliencia de los y las educadoras sociales; 2 ) examinar las diferencias en relación con el bienestar emocional y la resiliencia según diversas variables sociodemográficos; y 3) analizar la relación entre el bienestar emocional y la resiliencia en este grupo de profesionales.

\section{Método}

Se presenta una investigación cuantitativa de tipo exploratorio, descriptivo, transversal y correlacional (Montero y León, 2007; Vicencio, 2018), mediante un diseño ex post facto (Ary et al., 2010; Ato et al., 2013).

\section{Participantes}

En el estudio participaron 72 profesionales de la educación social (30.6\% varones, $69.4 \%$ mujeres) del ámbito residencial de protección a la infancia y la adolescencia de Cataluña: centro de acogida (CA), centro residencial de acción educativa (CRAE), centro residencial de educación intensiva (CREI), pisos de emancipación para mayores de edad (PE), y residencias para jóvenes sin referentes familiares (SRF).

La muestra fue seleccionada mediante muestreo no-probabilístico tipo bola de nieve (Hernández-Sampieri y Mendoza, 2018), un muestreo que ha resultado útil cuando previamente existe dificultad para acceder al escenario (Ruiz, 2008).

Los criterios de selección fueron: a) ser profesional de la intervención socioeducativa en el ámbito residencial de protección a la infancia, y b) haber estado en activo durante el período de confinamiento domiciliario. 
En la Tabla 1 se desglosan los estadísticos descriptivos referentes a la edad, estado civil, años de experiencia, formación, recurso residencial y supervisión, según el género de las personas participantes. La edad media es de 36.29 años (DT=9.66). El 63.9\% de las personas participantes mantiene una relación de pareja estable. La formación académica es mayoritariamente universitaria (80.6\%). La experiencia profesional se sitúa alrededor de los 10 años de media. La institución residencial más representada es el centro residencial de acción educativa, CRAE (72.2\%). Finalmente, se señala que el $86.1 \%$ cuenta con espacios de supervisión de la práctica profesional.

\section{Tabla 1}

Estadísticos descriptivos de los datos sociodemográficos

\begin{tabular}{|c|c|c|c|c|c|c|}
\hline \multirow{3}{*}{ Participantes } & \multicolumn{2}{|c|}{ Varones } & \multicolumn{2}{|c|}{ Mujeres } & \multicolumn{2}{|c|}{ Total } \\
\hline & $\mathrm{n}$ & $\%$ & $\mathrm{n}$ & $\%$ & \multirow{2}{*}{\multicolumn{2}{|c|}{$\begin{array}{c}n \\
72\end{array}$}} \\
\hline & 22 & 30.6 & 50 & 69.4 & & \\
\hline \multirow[t]{2}{*}{ Edad } & $M$ & $D T$ & $M$ & $D T$ & $M$ & $D T$ \\
\hline & 39.36 & 10.11 & 34.94 & 9.24 & 36.29 & 9.96 \\
\hline Estado civil & $n$ & $\%$ & $\mathbf{n}$ & $\%$ & $\mathrm{n}$ & $\%$ \\
\hline Soltera/o & 5 & 22.7 & 15 & 30.0 & 20 & 27.8 \\
\hline Casada/o & 8 & 36.4 & 4 & 8.0 & 12 & 16.7 \\
\hline Separada/o & 1 & 4.5 & 2 & 4.0 & 3 & 4.2 \\
\hline En convivencia & 8 & 36.4 & 26 & 52.0 & 34 & 47.2 \\
\hline No contesta & 0 & 0.0 & 3 & 6.0 & 3 & 4.2 \\
\hline \multirow[t]{3}{*}{ Experiencia profesional } & $M$ & $D T$ & $M$ & $D T$ & $M$ & $D T$ \\
\hline & 14 & 11 & 9 & 8 & 10.56 & 8.98 \\
\hline & $n$ & $\%$ & $\mathbf{n}$ & $\%$ & $\mathrm{n}$ & $\%$ \\
\hline Entre 1 y 2 años & 1 & 4.5 & 7 & 14.0 & 8 & 11.1 \\
\hline Entre 3 y 6 años & 8 & 35.4 & 18 & 36.0 & 26 & 36.1 \\
\hline Entre 7 y 12 años & 4 & 18.2 & 10 & 20.0 & 14 & 19.4 \\
\hline$>12$ años & 9 & 40.9 & 15 & 30.0 & 24 & 33.3 \\
\hline Formación & $\mathrm{n}$ & $\%$ & $\mathrm{n}$ & $\%$ & $\mathrm{n}$ & $\%$ \\
\hline CGSIS & 0 & 0.0 & 6 & 12.0 & 6 & 8.3 \\
\hline ES & 18 & 81.8 & 40 & 80 & 58 & 80.6 \\
\hline Habilitación & 3 & 13.6 & 4 & 8.0 & 7 & 9.7 \\
\hline Otros & 1 & 4.5 & 0 & 0.0 & 1 & 1.7 \\
\hline Institución & $n$ & $\%$ & $\mathbf{n}$ & $\%$ & $\mathrm{n}$ & $\%$ \\
\hline CA & 2 & 9.1 & 6 & 12.0 & 8 & 11.1 \\
\hline CRAE & 18 & 81.8 & 34 & 68.0 & 52 & 72.2 \\
\hline CREI & 1 & 4.5 & 1 & 2.0 & 2 & 2.8 \\
\hline $\mathrm{PE}$ & 1 & 4.5 & 2 & 4.0 & 3 & 4.2 \\
\hline SRF & 0 & 0.0 & 7 & 14.0 & 7 & 9.7 \\
\hline Supervisión & $n$ & $\%$ & $\mathrm{n}$ & $\%$ & $\mathrm{n}$ & $\%$ \\
\hline Sí & 17 & 77.3 & 45 & 90.0 & 62 & 86.1 \\
\hline No & 5 & 22.7 & 5 & 10.0 & 10 & 13.9 \\
\hline
\end{tabular}

Nota: Para la edad y los años de experiencia profesional, los datos reflejan la media y la desviación típica, junto con el porcentaje según bloques de experiencia profesional; el estado civil se segrega en personas solteras y sin relación estable, casadas, separadas/divorciadas o sin pareja estable, convivencia estable o pareja de hecho; la formación académica se distribuye entre el ciclo de grado superior de integración social, la diplomatura/grado universitario de educación social, la habilitación, y otras titulaciones; y la existencia de espacios de supervisión de la práctica educativa 


\section{Instrumentos}

En cuanto a los instrumentos utilizados, se recogió información relativa a diferentes variables sociodemográficas (edad, sexo/género, estado civil, experiencia profesional, formación, centro de trabajo y existencia de espacios de supervisión de la práctica profesional) además de los criterios para los afectos positivos y negativos, satisfacción con la vida y resiliencia, con el propósito de analizar las diferencias significativas en función de estas.

Escala de Afecto Positivo y Negativo (PANAS) (Watson et al., 1988; adaptación española de López-Gómez et al., 2015). Evalúa el componente afectivo del bienestar emocional. La escala está formada por 20 ítems, 10 para evaluar el Afecto Positivo (AP), y 10 para el Afecto Negativo (AN). Señala el grado en que las personas sienten las emociones, empleando una escala Likert de 1 ("Nada o muy ligeramente") a 5 ("Mucho"). El Balance Afectivo se obtiene de restar la puntuación de AN a la de AP; de manera que una puntuación positiva indicaría el predominio del afecto positivo; y una puntuación negativa indicaría el predominio del afecto negativo (Sanjuán y Ávila, 2016). En el presente estudio, la escala muestra una fiabilidad alfa de Cronbach de .89 para el Afecto Positivo, y de .88 para el Afecto Negativo.

Escala de Satisfacción con la Vida (SWLS) (Diener et al., 1985; adaptación española de Atienza et al. (2000). Evalúa el componente de satisfacción con la vida del bienestar emocional Consta de 5 ítems, en una escala Likert de 1 ("Muy en desacuerdo") a 5 ("Muy de acuerdo"), que recogen la valoración global que las personas hacen sobre su grado de satisfacción con la vida. La fiabilidad alfa de Cronbach en este estudio es .78.

Escala de Resiliencia (RS-14) (Wagnild y Young; 1993; adaptación española de SánchezTeruel y Robles-Bello, 2015). Evalúa la resiliencia mediante una puntuación unifactorial, recogiendo el acuerdo o desacuerdo en relación con 14 ítems, en una escala Líkert de 1 ("Totalmente en desacuerdo") a 7 ("Totalmente de acuerdo"). En esta investigación, la escala muestra una fiabilidad alfa de Cronbach de .86 .

Encuesta Sociodemográfica. Se diseñó una breve encuesta para recoger datos sociodemográficos relativos a edad, género, estado civil, experiencia profesional en medio residencial, recurso residencial y existencia de supervisión educativa.

\section{Procedimiento}

Se contactó con la dirección de diferentes recursos residenciales a través de correo electrónico, después de la finalización del estado de alarma y el confinamiento domiciliario. A través de este email, estas personas clave facilitaron la distribución de un enlace para que las 
educadoras y educadores sociales participantes pudieran acceder a la encuesta sociodemográfica y las tres escalas (mediante un formulario online). Las personas participantes fueron informadas por escrito acerca de la finalidad del estudio y se contó con su consentimiento informado. Se tuvo en cuenta la Declaración de Helsinki, así como el Código Europeo de Conducta para la Integridad en la Investigación (ALLEA, 2018) en la elaboración del estudio: consentimiento informado por escrito, participación voluntaria en el estudio, y garantía de anonimato y confidencialidad. También se ha atendido a las recomendaciones de Barbor et al. (2017) y Casado y Puigdoménech (2018) en la redacción de este artículo: ausencia de conflicto de intereses por parte del equipo investigador, y rigor y transparencia en la investigación y la comunicación de los resultados.

Por otra parte, las personas informantes clave hicieron llegar a la autora y autores del estudio unas observaciones acerca de la participación de las educadoras y educadores sociales que habían estado en activo durante el confinamiento, señalando su agotamiento físico y mental y la posibilidad de que esto afectase al número de participantes.

En cuanto al análisis de los datos recogido, se realizó un análisis descriptivo, que permitió establecer la distribución de frecuencias, medidas de tendencia central y medidas de variabilidad. Se llevó a cabo la comparación de las diferentes variables objeto de estudio (bienestar emocional y resiliencia) y los parámetros sociodemográficos, mediante las pruebas no-paramétricas $U$ de Mann-Withney y $H$ de Kruskal-Wallis; para obtener medidas de asociación se calculó la correlación de Spearman. El análisis de datos se realizó utilizando el programa IBM SPSS (versión 25).

\section{Resultados}

\section{Consistencia interna de las escalas y correlación entre Bienestar Subjetivo y Resiliencia}

En la Tabla 2 se muestran datos relativos a la consistencia interna de los instrumentos, y los estadísticos descriptivos de las variables de estudio: puntuaciones medias, desviaciones típicas y valores mínimos y máximos de Afectos Positivos y Negativos, Balance Afectivo, Satisfacción con la Vida (variables implicadas en el Bienestar Emocional) y Resiliencia.

La fiabilidad de las escalas utilizadas en este estudio no difiere de la encontrada en la población española por López-Gómez et al. (2015) para la PANAS, Atienza et al. (2020) para la SWLS, o Sánchez-Teruel y Robles (2015) para la RS-14. Esto permite constatar la bondad de los instrumentos para este estudio (Rodríguez y Reguant, 2020). 
Tabla 2

Estadísticos descriptivos y correlaciones de las variables Bienestar Subjetivo y Resiliencia

\begin{tabular}{lcccccccc}
\hline Variable & $M$ & $D T$ & Min & Máx & AP & AN & BA & SV \\
\hline Afecto Positivo (AP) & 33.93 & 6.69 & 17 & 50 & - & & & \\
Afecto Negativo (AN & 24.71 & 7.49 & 10 & 42 & $-.471^{* *}$ & - & & \\
Balance Afectivo (BA) & 9.22 & 11.88 & -17 & 40 & $.810^{* *}$ & $-.880^{* *}$ & - & \\
Satisfacción con la Vida (SV) & 18.79 & 3.24 & 10 & 25 & $.532^{* *}$ & $-.385^{* *}$ & $.531^{* *}$ & - \\
Resiliencia (R) & 78.78 & 8.79 & 52 & 98 & $.450^{* *}$ & $-.234^{*}$ & $.369^{* *}$ & $.366^{* *}$ \\
\hline
\end{tabular}

$* p<.05 ; * * p<.01$

El estadístico rho de Spearman señala la existencia de una correlación negativa entre Afecto Positivo y Afecto Negativo (-.451), y una correlación positiva con Balance Afectivo (.790), Satisfacción con la Vida (.439) y Resiliencia (.482). El Afecto Negativo tiene una correlación negativa con el Balance Afectivo (-.888), la Satisfacción con la Vida (-.296) y la Resiliencia (-.353).

El Balance Afectivo tiene una correlación positiva con la Satisfacción con la Vida (.412) y la Resiliencia (.485). Finalmente señalar la correlación positiva entre Satisfacción con la Vida y Resiliencia (.342).

\section{Distribución y comparación del bienestar subjetivo y resiliencia según las variables sociodemográficas}

Para análisis de las diferencias en función del género, se utilizó la prueba no paramétrica U de Mann-Whitney para dos muestras independientes (véase Tabla 3). Aunque las educadoras sociales obtienen mayores puntuaciones, no existen diferencias significativas entre las variables de PANAS, SWLS y RS-14.

\section{Tabla 3}

Medias y desviaciones típicas en función del género

\begin{tabular}{lcccc}
\hline & \multicolumn{2}{c}{ Masculino } & \multicolumn{2}{c}{ Femenino } \\
Variable & $M$ & $D T$ & $M$ & $D T$ \\
\hline Afecto Positivo & 32.91 & 5.35 & 34.38 & 7.21 \\
Afecto Negativo & 25.41 & 8.22 & 24.40 & 7.21 \\
Balance Afectivo & 7.50 & 11.74 & 9.98 & 11.98 \\
Satisfacción con la Vida & 18.00 & 3.22 & 19.14 & 3.22 \\
Resiliencia & 77.36 & 9.61 & 79.40 & 8.44 \\
\hline
\end{tabular}

Con relación al análisis de las diferencias en función de los años de experiencia profesional (véase Tabla 4), se determinaron cuatro intervalos (1-2 años, 3-6 años, 7-12 años y >12 años), realizándose la prueba no paramétrica $H$ de Kruskal Wallis. No se encontraron diferencias significativas en las variables analizadas. 


\section{Tabla 4}

Medias y desviaciones típicas en función de la experiencia profesional, expresada en años

\begin{tabular}{lcccccccc}
\hline & \multicolumn{2}{c}{$1-2$ años } & \multicolumn{2}{c}{$3-6$ años } & \multicolumn{2}{c}{$7-12$ años } & \multicolumn{2}{c}{$>12$ años } \\
Variable & $M$ & $D T$ & $M$ & $D T$ & $M$ & $D T$ & $M$ & $D T$ \\
\hline Afecto Positivo & 32.38 & 9.19 & 34.04 & 5.57 & 31.79 & 6.62 & 35.58 & 6.90 \\
Afecto Negativo & 28.28 & 8.71 & 25.00 & 6.96 & 23.00 & 6.51 & 24.00 & 8.01 \\
Balance Afectivo & 3.50 & 13.23 & 9.04 & 9.60 & 8.79 & 11.45 & 11.58 & 13.82 \\
Satisfacción con la Vida & 18.63 & 2.97 & 18.69 & 3.72 & 19.21 & 3.78 & 18.71 & 2.54 \\
Resiliencia & 76.13 & 5.89 & 79.62 & 9.12 & 77.86 & 8.69 & 79.29 & 9.54 \\
\hline
\end{tabular}

Con relación al estado civil, agrupado de manera dicotómica (con/sin relación de pareja estable), la formación académica agrupada dicotómicamente (formación universitaria/nouniversitaria), y con la participación en espacios de supervisión, se utilizó la prueba no paramétrica $U$ de Mann-Whitney Los resultados indican que no existen diferencias significativas entre las variables estudiadas y las variables sociodemográficas estado civil, formación académica y supervisión educativa.

Finalmente, se analizaron las diferencias en función de la institución residencial mediante la prueba no paramétrica $H$ de Kruskal Wallis (véase Tabla 5).

\section{Tabla 5}

Diferencias en las medias en función de la institución residencial (H de Kruskal-Wallis)

\begin{tabular}{lccccccccccc}
\hline & \multicolumn{2}{c}{ CA } & \multicolumn{2}{c}{ CRAE } & \multicolumn{2}{c}{ CREI } & \multicolumn{2}{c}{ PE } & \multicolumn{2}{c}{ SRF } & $H$ \\
Variable & $M$ & $D T$ & $M$ & $D T$ & $M$ & $D T$ & $M$ & $D T$ & $M$ & $D T$ & \\
\hline Afectos Positivos & 31.00 & 3.78 & 34.77 & 6.50 & 38.00 & 1.41 & 38.67 & .57 & 27.86 & 8.74 & 8.94 \\
Afectos Negativos & 27.63 & 4.92 & 23.69 & 7.69 & 32.50 & 7.77 & 19.67 & .57 & 28.86 & 7.01 & $10.15^{*}$ \\
Balance Afectivo & 3.37 & 5.97 & 11.08 & 12.49 & 5.50 & 9.19 & 19.00 & .00 & -1.00 & 6.32 & $11.05^{*}$ \\
Satisfacción Vida & 18.25 & 2.43 & 18.65 & 3.46 & 20.00 & 1.41 & 23.33 & 1.15 & 18.14 & 1.67 & 6.98 \\
Resiliencia & 77.00 & 6.14 & 78.87 & 9.52 & 88.00 & 1.41 & 78.00 & 6.93 & 77.86 & 7.19 & 4.75 \\
\hline
\end{tabular}

$* p<.05$

Los resultados señalan diferencias estadísticamente significativas en relación con las dimensiones Afecto Negativo y Balance Afectivo. Un análisis más detallado de las diferencias, mediante $U$ de Mann-Whitney para la dimensión Afecto Negativo, señala las diferencias significativas entre entro de acogida (CA) y pisos para mayores de 18 años (PE), o entre estos y los recursos para jóvenes sin referentes familiares (SFR), y entre centro residencial de educación intensiva (CREI) y pisos para mayores de 18 años (PE). Y para la dimensión Balance Afectivo, se encuentran diferencias significativas entre los recursos para jóvenes sin referentes familiares (SRF) centros residenciales de acción educativa (CRAE), entre recursos para jóvenes sin referentes familiares (SRF) y pisos para mayores de 18 años (PE), y centro de acogida (CA) y pisos para mayores de 18 años (PE). 


\section{Discusión}

El objetivo de este estudio era indagar el impacto de la COVID-19 y el confinamiento domiciliario en el bienestar emocional y la resiliencia de un grupo de educadoras y educadores sociales que trabajan en el ámbito residencial de protección a la infancia y adolescencia.

Los resultados evidencian que la muestra de este estudio es muy feminizada, con $69.4 \%$ de educadoras sociales. Estos resultados están en consonancia con los datos aportados por Estévez et al. (2017), el informe del estado de la educación social en Catalunya (CEESC, 2017) y el informe sobre la situación de los centros y recursos del sistema de protección de Catalunya (CEESC, 2019). Esto puede significar mayores dificultades en la conciliación de la vida personal y laboral porque, como ponen de manifiesto Altuzarra et al. (2018), en muchas ocasiones la conciliación sigue siendo una cuestión de la mujer, que asume una segunda jornada laboral. Este hecho puede contribuir a aumentar, por tanto, la sobrecarga laboral y el estrés en la profesión.

Se ha encontrado una correlación positiva entre el Balance Afectivo, la Satisfacción con la Vida y la Resiliencia en las educadoras y educadores sociales. Es decir, que cuando se da una puntuación alta en Balance Afectivo, también se da en Satisfacción con la Vida y en Resiliencia. Cuando aumenta una, aumenta la otra, si bien esta correlación no implica causalidad, es decir, no puede establecerse una relación de causa-efecto a partir de los resultados de esta correlación, si bien las y los autores habitualmente referenciados señalan que conceptualmente, el bienestar emocional es un factor resiliente. Estos datos muestran el papel de las emociones positivas en la resiliencia (Navarro et al., 2013). También consideran la resiliencia como un elemento clave para el afrontamiento, como señalan Aguaded y Almerida (2016), Dorado et al. (2021) y Vizoso (2019), y sugieren una relación positiva entre la regulación emocional y la satisfacción con la vida (Limonero et al, 2012), o la importancia de estos factores protectores frente al estrés laboral y el burnout, como señalan Heliz et al. (2015) o Navarro et al. (2018). Otros estudios señalan el papel protector de la resiliencia frente al confinamiento (Lozano-Díaz et al., 2020; Sánchez-Teruel et al., 2021). Todas estas evidencias coinciden con lo aportado por Wagnild (2013), quien recoge los resultados de diferentes estudios que señalan el papel protector de la resiliencia frente a la depresión, el estrés percibido y la ansiedad. Un elevado grado de bienestar emocional y de resiliencia resulta un factor protector de la salud mental (Asensio et al., 2017). Esta característica protectora del bienestar emocional se ha evidenciado durante la pandemia por la COVID-19 (Pérez-Fuentes, et al., 2021; Sánchez-Teruel et al., 2021).

Los resultados de este estudio y las evidencias procedentes de otras investigaciones ponen de manifiesto algunas orientaciones. En primer lugar, atender al bienestar emocional de 
la profesión (Dorado et al., 2021; Pons y González, 2012), dado el papel protector del bienestar emocional en la capacidad de afrontamiento resiliente. En segundo lugar, incluir el enfoque resiliente (Fernández de Araújo et al., 2015; Truter et al., 2017) en la práctica socioeducativa, que atienda no solamente a las competencias profesionales interpersonales y contextuales, sino también la perspectiva ecológica (Vaquero et al., 2014; Ungar, 2011). En tercer lugar, trabajar estrategias de regulación emocional para prevenir el burnout (Esteban-Ramiro y FernándezMontaño, 2017). En cuarto lugar, potenciar las prácticas de cuidado personal (Griffiths et al., 2019), dentro y fuera del entorno laboral, atendiendo a la importancia del cuidado de la persona. Por último, mejorar las relaciones interpersonales en el ámbito residencial teniendo en cuenta el factor protector que ejerce el soporte social laboral (Brend y Collin-Vézina, 2021) tanto formal (espacios de supervisión grupal) como informal (especialmente el apoyo de los iguales). A pesar de ser una profesión esencial, no está adecuadamente visibilizada. Por tanto, sumado a todo lo anterior, conviene mejorar las condiciones laborales, la capacitación académica y profesional (acorde a los cambios sociales), y el cuidado del bienestar emocional de las y los profesionales.

En cuanto a las limitaciones del estudio, esta es una investigación de carácter exploratorio, una aproximación al impacto de la pandemia y el confinamiento en un colectivo profesional específico como son las educadoras y educadores sociales. Esto implica cierta prudencia a la hora de generalizar resultados, pero posibilita una aproximación a las necesidades del colectivo profesional, y abre una vía para mejorar la acción socioeducativa en un contexto de alta complejidad y en una situación nueva e impredecible como ha sido el confinamiento (y con un futuro incierto frente a la posibilidad de nuevos rebrotes). Como futuras líneas de investigación, especialmente en el ámbito de la atención residencial a la infancia y la adolescencia, sería conveniente aumentar el tamaño de la muestra y estudiar longitudinalmente el bienestar emocional de estos profesionales después de la pandemia.

\section{Referencias}

AGUADED, María Cinta; \& ALMERIDA, Nora Alejandra (2016). La resiliencia del docente como factor crucial para superar las adversidades en una sociedad de cambios. Tendencias Pedagógicas, 28, 167-179. https://doi.org/10.15366/tp2016.28.012

ALLEA (2018). Código Europeo de Conducta para la Integridad en la Investigación. Berlín.

ALTUZARRA, Amaia; GÁLVEZ, Catalina; \& GONZÁLEZ, Ana María (2018). Diferencias de género en la distribución del tiempo de trabajo en las regiones españolas. Revista Internacional de Sociología, 76(3), e105. https://doi.org/10.3989/ris.2018.76.3.16.161 
ARY, Donald; JACOBS, Lucy; \& SORENSEN, Chris (2010). Introduction to Research in Education. Wadworth Cengage Learning.

ASENSIO, Angela; MAGALLÓN, Rosa; \& García, Javier (2017). Resiliencia: impacto positivo en la salud física y mental. Revista INFAD de Psicología, 2(2), 231-242. https://doi.org/10. 17060/ijodaep.2017.n2.v2.1096

ATIENZA, Francisco Luís; PONS, Diana; BALAGUER, Isabel; \& GARCÍA-MERITA, Marisa (2000). Propiedades psicométricas de la Escala de Satisfacción con la Vida en adolescentes. Psicothema, 12(2), 314-319. http://www.psicothema.com/psicothema.asp?id=296

ATO, Manuel; LÓPEZ, Juan; \& BENAVENTE, Ana (2013). Un sistema de clasificación de los diseños de investigación en psicología. Anales de psicología, 29(3), 1038-1059. https://doi.org/ 10.6018/analesps.29.3.178511

Banco de España (2020). Escenarios macroeconómicos de referencia para la economía española tras el COVID-19. Boletín económico del Banco de España, 2, 1-33. https://dialnet.unirioja.es/servlet/articulo?codigo=7622941

BABOR, Thomas; MCGOVERN, Thomas; \& ROBAINA, Katherine. (2017). Dante's Inferno: Seven Deadly Sins in Scientific Publishing and How to Avoid Them. En: Thomas Babor, Kerstin Stenius, Richard Pates, Michal Miovský, Jean. O’Reilly; \& Paul Candon (eds.), Publishing Addiction Science: A Guide for the Perplexed, (267-298). Ubiquity Press.

BREND, Denise; \& COLLIN-VÉZINA, Delphine (2021). Stronger together: Workplace social support among residential child welfare professionals. Child Abuse; \& Neglect. https://doi.org/ 10.1016/j.chiabu.2021.105302

CAMPOS, Miryam; IRAURGUÍ, Joseba; Páez, Darío; \& Velasco, Carmen (2004). Afrontamiento y regulación emocional de hechos estresantes. Un meta-análisis de 13 estudios. Boletín de Psicología, 82, 25-44. https://dialnet.unirioja.es/servlet/articulo?codigo=1302317

CASADO, María; \& PUIGDOMÈNECH, Pere (coords.) (2018). Documento sobre los aspectos éticos del diálogo entre ciencia y sociedad. Observatori de Bioètica i Dret, Universitat de Barcelona. http://www.edicions.ub.edu/refs/observatoribioeticadret/ficha.aspx?cod=08850

CASTRO, Alejandro (2009). El bienestar psicológico: cuatro décadas de progreso. Revista Interuniversitaria de Formación del Profesorado, 23(66), 43-72. https://dialnet. unirioja.es/servlet/articulo?codigo=3098202

CEESC - Col-legi d'Educadores i Educadors Socials de Catalunya (2017). L'Educació Social a Catalunya. CEESC. Disponible en: https://ceesc.cat/documents-publicacions/informesprofessionals/1022-informees-2017 
CEESC - Col-legi d'Educadores i Educadors Socials de Catalunya (2019). La situació professional i laboral als diferents centres i recursos del sistema de protección a la infància $i$ I'adolescència de Catalunya. CEESC. Disponible en: https://ceesc.cat/documentspublicacions/informes-professionals/1021-informe-infancia-2019-2

CEESC - Col-legi d'Educadores i Educadors Socials de Catalunya (2020). L'Educació Social enfront i després de la COVID-19. CEESC. Disponible en: https://ceesc.cat/documentspublicacions/informes-professionals/1052-informe-covid-2020

DE JUANAS, Ángel; LIMÓN, Rosario; \& MELENDRO, Miguel (2014). Práctica profesional y formación del educador social en España. Interfaces Científicas, 3(1), 89-102. https://doi.org/10.17564/2316-3828.2014v3n1p89-102

DIENER, Ed; EMMONS, Robert; LARSEN, Randy; \& GRIFFIN, Sharon (1985). The Satisfaction With Life Scale, Journal of Personality Assessment, 49(1), 71-75. https://doi.org/10.1207/ s15327752jpa4901_13

DORADO, Ana; PÉREZ, Jesús; RODRÍGUEZ-BRIOSO; María del Mar; \& GALLARDO, Lorena (2021). Emotional well-being and resilience during the COVID-19 pandemic: Guidelines for social work practice. International Social Work, 64(2), 279-284. https://doi.org/10.1177/ 0020872820970622

ESTEBAN-RAMIRO, Beatriz; \& FERNÁNDEZ-MONTAÑO, Patricia (2017). Las competencias para el desempeño profesional en Trabajo Social dentro de la formación: del burnout al engagement a través del manejo de las emociones. Trabajo Social Global. Revista de Investigaciones en Intervención social, 7(13), 142-168. https://doi.org/10.30827/tsggsw.v7i13.5839

ESTÉVEZ, Laura; FERNÁNDEZ, María Reyes; \& DAPÍA, María D. (2017). Animación sociolaboral y educación social: perfil profesional en el municipio de Ourense. Revista de Estudios e Investigación en Psicología y Educación, Extr.(14), 022-026 https://doi.org/10.17979/ reipe.2017.0.14.2241

FERNÁNDEZ-ABASCAL, Enrique; \& PALMERO, Francesc (1999). Emociones y salud. En: Enrique Fernández-Abascal y Francesc Palmero (coord.). Emociones y salud (pp. 5-18). Ariel.

FERNÁNDEZ DE ARAÚJO, Ludgleydson; TEVA, Inmaculada; \& BERMúDEZ, María Paz (2015). Resiliencia en adultos: una revisión teórica. Terapia Psicológica, 33(3), 257-276. https://teps.cl/index.php/teps/article/view/11

FERNÁNDEZ DEL VALLE, Jorge (2009). Evolución histórica, modelos y funciones del acogimiento residencial. En: Amaia Bravo y Jorge Fernández del Valle, Intervención socioeducativa en acogimiento residencial (pp. 11-24). Gobierno de Cantabria. 
FERNÁNDEZ DEL VALLE, Jorge; LÓPEZ, Mónica; \& BRAVO, Amaia (2007). Job stress and burnout in residential child care workers in Spain. Psicothema, 19(4), 610-615. http://www.psicothema.com/psicothema.asp?id=3405

Fondo Monetario Internacional (2021). Informe sobre la estabilidad financiera mundial, abril de 2021. Disponible en: https://www.imf.org/es/Publications/GFSR/Issues/2021/04/06/ global-financial-stability-report-april-2021\#ResumenEjecutivo

GARCÍA-MÍNGUEZ, Jesús; \& SÁNCHEZ-RAMOS, Juan Manuel (2010). Centros de día de atención a menores: competencias del educador social como figura de referencia. Revista Historia de la Educación Latinoamericana, 15, 125-146. https://doi.org/10.19053/01227238.1568

GRIFFITHS, Austin; ROYSE, David; MURPHY, April; \& STARKS, Saundra. (2019). Self-Care Practice in Social Work Education: A Systematic Review. of Interventions. Journal of Social Work Education, 55(1), 102-114. https://doi.org/10.1080/10437797.2018.1491358

HERNÁNDEZ SAMPIERI, Roberto; \& MENDOZA, Cristina Paulina (2018). Metodología de la investigación: las rutas cuantitativa, cualitativa y mixta. McGraw-Hill.

HELIZ, Jorge; NAVARRO, Ignasi; TORTOSA, Noelia; \& JODRA, Pablo (2015). Análisis de diferentes factores de riesgo de la profesión de educador en diferentes contextos residenciales de la provincia de Alicante. Revista sobre la infancia y la adolescencia, 9, 47-59. https://doi.org/10.4995/reinad.2015.3877

INFANTE, Francisca (2001). La resiliencia como proceso. En: Aldo Melillo; \& Elbio Néstor Suárez Ojeda (comp.), Resiliencia. Descubriendo las propias fortalezas (pp. 31-53). Paidós.

JENARO, Cristina; FLORES, Noelia; \& GONZÁLEZ-GIL, Francisca (2007). Síndrome de burnout y afrontamiento en trabajadores de acogimiento residencial de menores. International Journal of Clinical and Health Psychology, 7(1), 107-121. https://dialnet.unirioja.es/ servlet/articulo?codigo $=2251764$

KNIGHT, Cecily (2007). A resilience framework: perspectives for educators. Health Education, 107(6), 543-555. https://doi.org/10.1108/09654280710827939

LÁZARO, Araceli; ORTEGA, Daniel; PANCHÓN, Carmen; \& RODRÍGUEZ, Julio (2020). Informe. La figura de l'educadora i l'educador social a l'actual sistema català d'Atenció i de Protecció a la Infància i l'Adolescència. CEESC. Disponible en: https://ceesc.cat/documentspublicacions/informes-professionals/1019-document-cdp-2

LAZARUS, Richard; \& FOLKMAN, Susan (1986). Estrés y procesos cognitivos. Martínez Roca.

LIMONERO, Joaquín; TOMÁS-SÁBADO, Joaquín; FERNÁNDEZ-CASTRO, Jordi; GÓMEZ-ROMERO, Ma José; \& Ardilla-Herrero, Amor (2012). Estrategias de afrontamiento resilientes y 
regulación emocional: predictores de satisfacción con la vida. Behavioral Psychology/Psicología Conductual, 20(1), 183-196.

LÓPEZ-GÓMEZ, Irene; HERVÁS, Gonzalo; \& VÁZQUEZ, Carmelo (2015). Adaptación de la “Escala de afecto y negativo" (PANAS) en una muestra general española. Behavioral Pyschology /Psicología Conductual, 23(3), 529-548.

LOZANO-DÍAZ, Antonia; FERNÁNDEZ-PRADOS, Juan Sebastián; FIGUEREDO, Victoria; \& MARTíNEZ, Ana María (2020). Impactos del Confinamiento por el COVID-19 entre Universitarios: Satisfacción Vital, Resiliencia y Capital Social Online. International Journal of Sociology of Education, Special Issue, 79-104. https://doi.org/10.17583/rise.2020.5925

MANCIAUX, Michel; VANISTENDAEL, Stephan; LECOMTE, Jacques; \& CYRULNIK, Boris (2003). Conclusiones y perspectivas. En: Michel Manciaux (comp.), La resiliencia: resistir y rehacerse (pp. 303-318). Gedisa.

MARTíNEZ-PÉREZ, Alejandro; \& LEZCANO, FERNANDO (2020). Percepción del Impacto de la Covid-19 en los Profesionales de la Educación Social que Trabajan con Menores. Revista Internacional de Educación para la Justicia Social, 9(3), 223-243. https://doi.org/10. 15366/riejs2020.9.3.012

MONTERO, Ignacio; \& LEÓN, Orfelio (2007). A guide for naming research studies in Pyschology. International Journal of Clinical and Health Psychology, 7(3), 847-862. https://www.redalyc.org/articulo.oa?id=33770318

MONTSERRAT, Carme; \& MELENDRO, Miguel (2017). ¿Qué habilidades y competencias se valoran de los profesionales que trabajan con adolescencia en riesgo de exclusión social? Análisis desde la acción socioeducativa. Educación XXI, 20(2), 113-135. https://doi.org/10. 5944/educxx1.19034

NAVARRO, Ana; BUENO, Belén; \& BUZ, José (2013). Bienestar emocional en la vejez avanzada: estudio comparativo por edad y género. Psychology, Society, \& Education, 5(1), 41-57. https://doi.org/10.25115/psye.v5i1.495

NAVARRO, Ignasi; LÓPEZ, Begoña; HELIZ, Jorge; \& REAL, Marta (2018). Estrés laboral, burnout i estrategias de afrontamiento en trabajadores que intervienen con menores en riesgo de exclusión social. Aposta. Revista de Ciencias Sociales, 78, 68-96. https://dialnet.unirioja. es/servlet/articulo?codigo $=6585738$

NICOLA, Maria; ALSAFI, Zaid; SOHRABI, Catrin; KERWAN, Ahmed; AL-JABIR, Ahmed; IOSIFIDIS, Christos; AGHA, Maliha; \& AGHA, Riaz (2020). The socio-economic implications of the coronavirus pandemic (COVID-19): A review. International journal of surgery, 78, 185193. https://doi.org/10.1016/j.ijsu.2020.04.018 
PÉREZ-FUENTES, Maria del Carmen; MOLERO, María del Mar; MARTOS, África; SIMÓN, María del Mar; \& GÁZQUEZ, José Luís (2021). Estado de ánimo y equilibrio afectivo de los españoles confinados por COVID-19: un estudio transversal. International Journal of Psychological Research, 14(1), 55-65. https://doi.org/10.21500/20112084.4765.

PONS, Juan de Pablos; \& GONZÁLEZ, Alicia (2012). El bienestar subjetivo y las emociones en la enseñanza. Revista Fuentes, 12, 69-92. https://revistascientificas.us.es/index.php/ fuentes/article/view/2468

Real Decreto 463/2020, de 14 de marzo, por el que se declara el estado de alarma para la gestión de la situación de crisis sanitaria ocasionada por el COVID-19. BOE, núm. 67: https://www.boe.es/eli/es/rd/2020/03/14/463/con

Real Decreto-ley 10/2020, de 29 de marzo, por el que se regula un permiso retribuido recuperable para las personas trabajadoras por cuenta ajena que no presten servicios esenciales, con el fin de reducir la movilidad de la población en el contexto de la lucha contra el COVID-19. BOE, núm. 87: https://www.boe.es/eli/es/rdl/2020/03/29/10/con

REPPOLD, Caroline; KAISER, Vanessa; ZANON, Cristian; HUTZ, Claudio; CASANOVA, Joana R.; \& ALMEIDA, Leandro S. (2019). Escala de Satisfacción con la Vida: evidencias de validez y fiabilidad en universitarios portugueses. Revista de Estudios e Investigación en Psicología y Educación, 6(1), 15-23. https://doi.org/10.17979/reipe.2019.6.1.4617

ROBLES-BELLO, María Auxiliadora; SÁNCHEZ-TERUEL, David; \& VALENCIA-NARANJO, Nieves (2020). Variables protecting mental health in the Spanish population affected by the COVID-19 pandemic. Current Psychology. https://doi.org/10.1007/s12144-020-01132-1

RODRÍGUEZ, Julio; \& REGUANT, Mercedes (2020). Calcular la fiabilidad de un cuestionario o escala mediante el SPSS: el coeficiente alfa de Cronbach. REIRE Revista d'Innovació $i$ Recerca en Educació, 13(2), 1-13. https://doi.org/10.1344/reire2020.13.230048

RUIZ, Antoni (2008). La muestra: algunos elementos para su confección. REIRE Revista d'Innovació i Recerca en Educació, 1, 75-88. https://doi.org/10.1344/reire2008.1.1117

RUTTER, Michael (2012). Resilience as a dynamic concept. Development and Psychopathology, 24, 335-344. https://doi.org/10.1017/S0954579412000028

RYFF, Carol; \& SINGER, Burton (2000). Interpersonal flourishing: A positive health agenda for the new millennium. Personality and Social Psychology Review, 4(1), 30-44. https://doi.org/ 10.1207/S15327957PSPR0401_4

SÁNCHEZ-TERUEL, David; \& ROBLES-BELLO, Maria Auxiliadora (2015). Escala de Resiliencia 14 ítems (RS-14): Propiedades psicométricas de la versión en español. Revista 
Iberoamericana de Diagnóstico y Evaluación, 2(40), 103-113. https://dialnet.unirioja.es/ servlet/articulo?codigo $=6474694$

SÁNCHEZ-TERUEL, Daniel; ROBLES-BELLO, María Auxiliadora; SARHANI-ROBLES, Mariam; \& SARHANI-ROBLES, Aziz (2021). Exploring resilience and well-being of family caregivers of people with dementia exposed to mandatory social isolation by COVID-19. Dementia, O(0), 1-16. https://doi.org/10.1177/14713012211042187

SANJUÁN, Pilar; \& ÁVILA, María (2016). Afrontamiento y motivación como predictores del bienestar subjetivo y psicológico. Revista de Psicopatología y Psicología Clínica, 21, 1-10. https://doi.org/10.5944/rppc.vol.21.num.1.2016.15401

SISTO, Antonella; VICINANZA, Flavia; LEONDINA, Laura; RICCI, Giovanna; TARTAGLINI, Daniela; \& TAMBONE, Vittoradolfo (2019). Towards a transversal definition of psychological resilience: A literature review. Medicina, 55(11). 745. https://doi.org/10.3390/medicina55110745

TRUTER, Elmien; FOUCHÉ, Ansie; \& THERON, Linda (2019). The resilience of child protection social workers: Are they at risk and if so, how do they adjust? A systematic meta-synthesis. British Journal of Social Work, 47, 846-863. https://doi.org/10.1093/bjsw/bcw048

UNGAR, Michael (2011). The social ecology of resilience: addressing contextual and cultural ambiguity of a nascent construct. American Journal of Orthopsychiatry, 81(1), 1-17. https://doi.org/10.1111/j.1939-0025.2010.01067.x

VALLÉS, Josep (2011). Análisis y valoración de las funciones de los educadores sociales en España [Tesis doctoral, Universidad Nacional de Educación a Distancia (UNED)]. Repositorio Institucional de la UNED e-spacio: http://e-spacio.uned.es/fez/view/tesisuned:Educacion -Jvalles

VANISTENDAEL, Stephan (2018). Hacia la puesta en práctica de la resiliencia. BICE.

VAQUERO, Eduard; URREA, Aida; \& MUNDET, Anna (2014). Promoting resilience through technology, art and child rights-based approach. Revista de cercetare si interventiesociala, 45, 144-159. http://hdl.handle.net/10459.1/48367

VÁZQUEZ, Carmelo; HERVÁS, Gonzalo; RAHONA, Juan José; \& GÓMEZ, Diego (2009). Bienestar psicológico y salud: Aportaciones desde la Psicología Positiva. Anuario de Psicología Clínica y de la Salud, 5, 15-28. https://dialnet.unirioja.es/servlet/articulo?codigo=3328166

VERA, Beatriz; CARBELO, Begoña; \& VECINA, María Luisa (2006). La experiencia traumática desde la psicología positiva: resiliencia y crecimiento postraumático. Papeles del Psicólogo, 27(1), 40-49. Disponible en: http://www.papelesdelpsicologo.es/contenido? num $=1120$ 
VICENCIO, Omar (2018). La investigación en las ciencias sociales. Trillas.

VIZOSO, Carmen (2019). Resiliencia, optimismo y estrategias de aforntamiento en estudiantes de Ciencias de la Educación. Psychology, Society, \& Education, 11(3), 367-377. https://doi.org/10.25115/psye.v11i3.2280

WALKER, Patrick; WHITTAKER, Charles; WATSON, Oliver; BAGUELIN; Marc; AINSLIE, Kylie; BHATIA, Sangeeta; BHATT, Samir; BOONYASIRI, Adhiratha; BOYD, Olivia; CATTARINO, Lorenzo; CUCUNUBÀ, Zulma; CUIMO-DANNEBURG, Gina et al. (2020). The Global Impact of COVID-19 and Strategies for Mitigation and Suppression - Spanish translation. Imperial College London. https://doi.org/10.25561/77735

WAGNILD, Gail (2013). Development and Use of the Resilience Scale (RS) with Middle -Aged and Older Adults. En: Sandra Prince-Embury; \& Donald Saklofske (eds.), Resilience in Children, Adolescents, and Adults: Translating Research into Practice (pp. 151-160). Springer Science+Business Media. Disponible en: https://link.springer.com/book/10.1007/978-14614-4939-3

WATSON, David; CLARK, Lee Anna; \& TELLEGEN, Auke (1988). Development and validation of brief measures of positive and negative affect: The PANAS scales. Journal of Personality and Social Psychology, 54(6), 1063-1070. https://doi.org/10.1037/0022-3514.54.6.1063

Fecha de recepción: 18 de septiembre de 2021. Fecha de revisión: 14 de noviembre de 2021. Fecha de aceptación: 16 de noviembre de 2021. Fecha de publicación: 1 de diciembre de 2021. 